NSF-ITP-00-112

ITEP-TH-37/00

\title{
Giant Gravitons from Holomorphic Surfaces
}

\author{
Andrei Mikhailov日 \\ Institute for Theoretical Physics, \\ University of California, Santa Barbara, CA 93106 \\ E-mail: andrei@itp.ucsb.edu
}

\begin{abstract}
We introduce a class of supersymmetric cycles in spacetimes of the form AdS times a sphere or $T^{1,1}$ which can be considered as generalizations of the giant gravitons. Branes wrapped on these cycles preserve $\frac{1}{2}, \frac{1}{4}$ or $\frac{1}{8}$ of the supersymmetry. On the CFT side these configurations correspond to superpositions of the large number of BPS states.
\end{abstract}

\footnotetext{
${ }^{1}$ On leave from the Institute of Theoretical and Experimental Physics, 117259, Bol. Cheremushkinskaya, 25, Moscow, Russia.
} 


\section{Introduction}

Supergravity on $A d S$ spaces has received a lot of attention in the last few years because of its conjectured relation to the Conformal Field Theory [1]. This relation implies the correspondence between the primary operators in the bulk and the supergravity fields propagating in the $A d S$ space. The standard way to formulate this correspondence goes as follows [2, 3]. Suppose that the Yang-Mills coupling constant $g_{Y M}^{2}$ is very small and the t'Hooft coupling constant $g_{Y M}^{2} N$ is very large, then the dual string theory on $A d S_{5} \times S^{5}$ should be well described by the classical supergravity. Upon the reduction of the massless fields in the classical supergravity on $S^{5}$ we get the series of massive fields in AdS, which correspond to the primary operators in the conformal field theory. The spectrum of the Kaluza-Klein excitations in AdS supergravities was computed in [4, 5, 6, 7], and this gives us the description of the spectrum of primary operators on the CFT side. But this description is good only for those operators which have relatively small conformal dimensions. Increasing the conformal dimension corresponds on the supergravity side to increasing the momentum of the Kaluza-Klein harmonic. When the momentum is high enough, it could happen that the linearized approximation to supergravity breaks down and we have to take into account interactions. Let us consider for example the four-dimensional $N=4$ supersymmetric Yang-Mills theory which is dual to the Type IIB supergravity on $A d S_{5} \times S^{5}$. The Kaluza-Klein harmonics are parametrized by their momentum $M$ which is an integer (strictly speaking, a set of integers). The energy of this harmonic is $m=\frac{M}{R}$, where $R$ is the radius of $A d S_{5} \times S^{5}$ (we assume that our harmonic saturates the BPS bound). We can interpret this energy as the mass of the corresponding five-dimensional field in $A d S_{5}$. The particle with such a mass has the Schwarzschild radius

$$
r_{S} \simeq \sqrt{\frac{g_{s t r}^{2}}{R^{5}} m}=\frac{1}{m} \sqrt{\frac{M^{3}}{N^{2}}}
$$

where we have taken into account $g_{s t r} N \simeq R^{4}$. We see that for $M>>N^{2 / 3}$ the Schwarzschild radius becomes larger then the Compton wavelength, which indicates the breakdown of the linearized supergravity approximation. Therefore the analysis of [4, 5, 6, 7] does not work for the high momentum states.

The supergravity description of the $\frac{1}{2}$ BPS states with high $M$ was found by McGreevy, Susskind and Toumbas [8]. They have found that these states should be considered not as Kaluza-Klein reductions of massless supergravity fields but as branes. This is an example of a general phenomenon: the massless particle can "polarize" and become a brane [9]. The branes representing the high momentum states were called giant gravitons.

Giant gravitons preserving half of the supersymmetries have been studied in [8, 10, 11. It has been found that they come in two species. The first type are spheres inside $S^{5}$, the projection of the worldvolume on the AdS space being a timelike geodesic. The second type are the spheres inside $A d S_{5}$, the projection on a sphere being an equator. (These two types are formally related by an analytic continuation, which relates $A d S_{5} \times$ 
$S^{5}$ to $S^{5} \times A d S_{5}$.) In our paper we discuss more general configurations preserving $\frac{1}{4}$ or $\frac{1}{8}$ supersymmetry. We present a class of such configurations parametrized by the holomorphic curves in $\mathbf{C}^{3}$. We formulate our results and explain the CFT meaning of our configurations in Section 2. In Sections 3,4 and 5 we prove that our configurations are supersymmetric. In Section 6 we derive the mass formula and verify the saturation of the BPS bound. In Section 7 we consider similar configurations in flat space. In Appendix we comment on the relation between our results and the results of the linearized approximation which was developed in [12].

\section{The construction}

\section{1 $\quad A d S_{5} \times S^{5}$ and $A d S_{5} \times T^{1,1}$}

Consider a five-dimensional manifold $M$ embedded into a six-dimensional $N$. In our application, $M$ is a part of the $1+9$-dimensional space-time of Type IIB string theory, and $N$ is an auxiliary manifold (the orthogonal direction to $M$ in $N$ does not belong to the space-time and does not have any physical meaning). For example, we can take $M=S^{5}$ of $A d S_{5} \times S^{5}$, and $N$ to be $\mathbf{R}^{6}$. Suppose that $N$ has a complex structure. In this situation, $M$ has a preferred field of directions. This field of directions is defined as follows. Let $e^{\perp}$ be the unit vector in $T N$ orthogonal to $T M$. Then, $e^{\|}=I . e^{\perp}$ belongs to the tangent space of $M$. This gives us the field of directions in $M$.

Given a holomorphic surface $C \subset N$, we associate to it a giant graviton. We will do it in the following way. Let us denote $\Sigma$ the intersection of $C$ with $M$. We will consider $\Sigma$ to be the surface of the $D 3$ brane at time $\tau=0$. Let us move this brane by translation in the preferred direction $e^{\|}$with the speed of light. Since $e^{\|}$is usually not orthogonal to $\Sigma$, the surface elements of the brane actually move with the speed less than the speed of light. We claim that the resulting $1+3$-dimensional worldvolume is supersymmetric.

Example. Let us consider $S^{5} \subset \mathbf{C}^{3}$. We denote $Z_{1}, Z_{2}, Z_{3}$ the flat coordinates on $\mathbf{C}^{3}$,

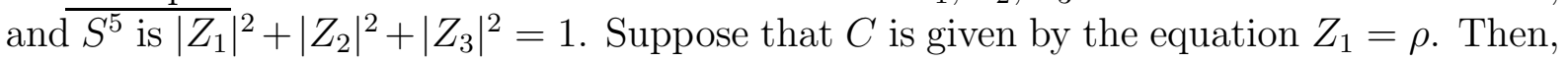
$\Sigma=S^{3}$, and the resulting worldvolume is the giant graviton found in $[\mathbb{Z}]$. The spatial trajectory of this giant graviton is $S^{1} \times S^{3}$.

More generally, we can consider $C$ described by the equation of the form $F\left(Z_{1}, Z_{2}, Z_{3}\right)=$ 0 , and the worldsurface of the giant graviton at time zero will be

$$
\left\{\begin{array}{l}
\left|Z_{1}\right|^{2}+\left|Z_{2}\right|^{2}+\left|Z_{3}\right|^{2}=1 \\
F\left(Z_{1}, Z_{2}, Z_{3}\right)=0
\end{array}\right.
$$

Suppose that we measure time as the length of the geodesic in AdS. Then, the worldsurface of the giant graviton at time $t$ will be:

$$
\left\{\begin{array}{l}
\left|Z_{1}\right|^{2}+\left|Z_{2}\right|^{2}+\left|Z_{3}\right|^{2}=1 \\
F\left(e^{-i t / R} Z_{1}, e^{-i t / R} Z_{2}, e^{-i t / R} Z_{3}\right)=0
\end{array}\right.
$$


where $R$ is the radius of $S^{5}$. This is a supersymmetric trajectory.

We can apply our construction for gravitons in $A d S_{5} \times T^{1,1}$. In this case, we should embed $T^{1,1}$ into the conifold, as explained in [14]:

$$
\begin{gathered}
X_{1}^{2}+\ldots+X_{4}^{2}=0 \\
\left|X_{1}\right|^{2}+\ldots+\left|X_{4}\right|^{2}=1
\end{gathered}
$$

The manifold $T^{1,1}$ is a $U(1)$ bundle over $S^{2} \times S^{2}$, and the preffered direction $e^{\|}$is in fact the direction along the fiber. Translation along this preffered direction corresponds to the phase rotation of $X_{I}$. The equation for the giant graviton is:

$$
\left\{\begin{array}{l}
X_{1}^{2}+\ldots+X_{4}^{2}=0 \\
\left|X_{1}\right|^{2}+\ldots+\left|X_{4}\right|^{2}=1 \\
F\left(e^{-i t / R} X_{1}, \ldots, e^{-i t / R} X_{4}\right)=0
\end{array}\right.
$$

We have not tried to turn on the gauge field living on the D3 brane.

\section{$2.2 \quad A d S_{3} \times S^{3}$}

The spacetime $A d S_{3}$ arises in the study of D-branes of Type IIB string theory on $\mathbf{T}^{4}$ or $K 3$. We will consider Type IIB on $\mathbf{T}^{4}$. D-branes with $1+1$ noncompact dimensions are effectively described as six-dimensional black strings. These black strings are classified by the charge $Z \in H^{*}\left(\mathbf{T}^{4}, \mathbf{Z}\right)$. Take a large number $N$ of black strings all having the same charge $Z$. The geometry of the near horizon region is $A d S_{3} \times S^{3} \times \mathbf{T}^{4}$. Consider the probe string having the same charge $Z$. Suppose that at the time $t=0$ the probe string spans a one-dimensional curve $\gamma \subset S^{3}$, and is a point in $A d S_{3}$. Let us think of $S^{3}$ as a group manifold of $S U(2)$. Suppose that our probe string moves along a geodesic line in $A d S_{3}$ and its position in $S^{3}$ changes as follows:

$$
\gamma \mapsto e^{i t \sum_{i=1}^{3} x_{i} \sigma^{i}} \cdot \gamma
$$

where $\sigma^{i}$ are Pauli matrices, $\sum_{i=1}^{3} x_{i}^{2}=1, t$ is the length parameter along the geodesic. In other words, we translate our effective string along the one-parameter subgroup in $S U(2)$ with the speed of light. We claim that this brane configuration is supersymmetric, preserving $\frac{1}{4}$ of the supersymmetries. We will prove it in Section 4 .

This gives us a class of supersymmetric configurations in $A d S_{3} \times S^{3}$ parametrized by the one-dimensional curves $\gamma \subset S^{3}$.

\subsection{Giant gravitons in $A d S_{4} \times S^{7}$ and $A d S_{7} \times S^{4}$}

The construction of giant gravitons for $A d S_{4} \times S^{7}$ is similar to the construction for $A d S_{5} \times$ $S^{5}$. We embed $S^{7}$ into $\mathbf{C}^{4}$ and consider intersections with the holomorphic surfaces in $\mathrm{C}^{4}$. We get configurations preserving $\frac{1}{2}, \frac{1}{4}$ and $\frac{1}{8}$ supersymmetries. 
Supersymmetric cycles in $A d S_{7} \times S^{4}$ can be constructed in the following way. We embed $S^{4} \subset \mathbf{R}^{5}$ and represent $\mathbf{R}^{5}=\mathbf{R} \times \mathbf{C}^{2}$. Then we consider the cylindrical surfaces in $\mathbf{R}^{5}$ of the form $\mathbf{R} \times C$ where $C \subset \mathbf{C}^{2}$ is a holomorphic curve. Intersection of these cylinders with $S^{4}$ gives us the worldvolumes of $M 2$ branes at time zero. The motion of the $M 2$ corresponds to multiplication of the coordinates of $\mathbf{C}^{2}$ by $e^{-i t}$. These configurations for generic $C$ preserve $\frac{1}{4}$ of the supersymmetries. If $C$ is a plane then we reproduce the giant graviton of $\left[\mathbb{8}\right.$ which preserves $\frac{1}{2}$ of the supersymmetry. We can interpret the splitting $\mathbf{R}^{5}=\mathbf{R} \times \mathbf{C}^{2}$ in the following way. The momentum of the BPS state is an element of $s o(5)$ and it can be represented as an antisymmetric matrix $M_{I J}$ acting in $\mathbf{R}^{5}$. An antisymmetric matrix acting in an odd-dimensional space has at least one zero eigenvector. The preffered direction $\mathbf{R}$ is generated by a vector annihilated by $M_{I J}$.

The details for $A d S_{4} \times S^{7}$ and $A d S_{7} \times S^{4}$ are presented in Section 5 .

\subsection{Giant Gravitons and BPS States}

We have seen that there is a continuum of supersymmetric giant gravitons with the given momentum, parametrized by the holomorphic curves (or just arbitrary curves, in the case of $A d S_{3} \times S^{3}$ ). One could think that this implies that there is a continuum of BPS states with a given momentum on the CFT side. Of course, this conclusion is wrong. To find the degeneracy of the BPS states one should study the giant gravitons quantum mechanically. Classical supersymmetric solutions of the D-brane equations of motion corresponding to the giant gravitons are in fact superpositions of the large number of supersymmetric quantum states having nearly the same energy and momentum. Let us think of the momentum $M$ as specifying an irreducible representation of the $R$-symmetry group (essentially, a set of integers). What is the number of states with the given momentum $M$ ? On the CFT side, we at least naively expect it to depend on $M$ and the number of colors $N: \mathcal{N}=\mathcal{N}(M, N)$ - it is the number of primary operators transforming in a given representation of the R-symmetry group. On the supergravity side $N$ is proportional $T R^{p+1}$ where $T$ is the tension of the probe brane (the brane which makes the giant graviton) and $R$ is the radius of the AdS space. The quantity $T R^{p+1}$ is in fact the only dimensionless parameter in the Born-Infeld action for the probe brane, if we forget about $\alpha^{\prime}$-corrections. Naively this implies that the number of states with the given momentum is a function of $T R^{p+1}$, which would agree with what we expect from CFT. But one should remember that the Born-Infeld action is not enough to define a worldvolume theory on a D-brane. There are divergences which should be regularized by adding $\alpha^{\prime}$ corrections. In fact, considering the full quantum theory on the D brane requires taking into account the degrees of freedom living in the bulk. Either the degeneracy of the BPS states of the $\mathrm{D}$ brane does not receive $\alpha^{\prime}$ corrections, or the number of primary operators in CFT depends on the coupling constant.

We will not try to quantize the worldvolume theory of the giant graviton in our paper. But we want to consider a simple and well-known example in flat space where we can 
actually compute the degeneracy.

Example. Classical BPS configurations of the D-string.

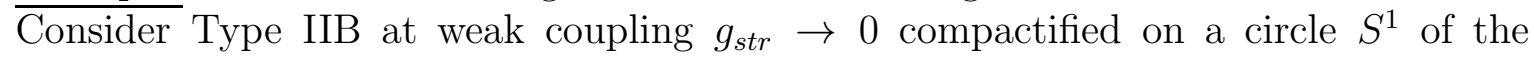
radius $R_{9}$. Let us wrap $D 1$ on $S^{1}$. Let $X^{0}$ be the time, $X^{9}$ the coordinate on the circle, and $X^{I}(\tau, \sigma), I=1, \ldots, 8$ the coordinates in the noncompact directions. Consider the following classical solution of the D-string equations of motion:

$$
X^{I}(\tau, \sigma)=f^{I}(\sigma+\tau)
$$

This is the left moving wave. This solution preserves $\frac{1}{4}$ of the supersymmetry of type IIB (we review this fact in Section 7). It saturates the BPS bound for a given momentum. Indeed, the energy of the solution is equal to:

$$
E=T \int d l \frac{1}{\sqrt{1-v_{\perp}^{2}}}=T \int \frac{d l^{2}}{d X^{9}}=2 \pi R_{9} T+T \int d X^{9} \sum_{I=1}^{8}\left(\frac{d X^{I}}{d X^{9}}\right)^{2}
$$

where $T$ is the tension of the D-string. We have taken into account that $v_{\perp}^{9}=\left(\frac{d X^{9}}{d l}\right)^{2}-1$, $v_{\perp}^{I}=\frac{d X^{9}}{d l} \frac{d X^{I}}{d l}$ and $\left(v_{\perp}\right)^{2}=1-\left(\frac{d X^{9}}{d l}\right)^{2}=\sum_{I=1}^{8}\left(\frac{d X^{I}}{d l}\right)^{2}$. The momentum along the circle is:

$$
P_{9}=T \int d l \frac{v_{\perp}^{2}}{\sqrt{1-v_{\perp}^{2}}}=T \int d X^{9} \sum_{I=1}^{8}\left(\frac{d X^{I}}{d X^{9}}\right)^{2}
$$

We see that the BPS bound is saturated:

$$
E=2 \pi R_{9} T+P_{9}
$$

Quantum mechanically $P_{9}$ should be quantized and for each integer $P_{9}$ there is a finite number of states. Let us briefly review how this happens. Suppose that the coordinates are slowly varying on the worldsheet:

$$
S \simeq-2 \pi R T \int d X^{0}\left[1-\frac{1}{4 \pi R} \int d X^{9}\left(\left\|\frac{\partial X^{I}}{\partial X^{0}}\right\|^{2}-\left\|\frac{\partial X^{I}}{\partial X^{9}}\right\|^{2}\right)\right]
$$

We will forget about fermions. Consider the Fourier decomposition of the worldsheet fields:

$$
X^{I}\left(X^{0}, X^{1}\right)=\sum_{k=-\infty}^{\infty} X_{k}^{I}\left(X^{0}\right) e^{i k X^{9} / R}
$$

The action can be rewritten in terms of the Fourier modes:

$$
S=-2 \pi R T \int d X^{0}\left[1-\frac{1}{2} \sum_{k=-\infty}^{\infty}\left(\frac{d X_{-k}^{I}}{d X^{0}} \frac{d X_{k}^{I}}{d X^{0}}-\frac{k^{2}}{R^{2}} X_{-k}^{I} X_{k}^{I}\right)\right]
$$


The Hamiltonian is:

$$
H=\frac{1}{R} \sum_{k>0}\left(\left\|a^{k I}\right\|^{2}+\left\|\tilde{a}^{k I}\right\|^{2}\right)+\text { const }
$$

where

$$
\begin{aligned}
& a^{k I}=\frac{1}{\sqrt{2}}\left(\frac{1}{\sqrt{2 \pi T}} P^{k I}+i k \sqrt{2 \pi T} X^{-k I}\right) \\
& \tilde{a}^{k I}=\frac{1}{\sqrt{2}}\left(\frac{1}{\sqrt{2 \pi T}} P^{k I}-i k \sqrt{2 \pi T} X^{-k I}\right)
\end{aligned}
$$

and $P^{k I}$ is the momentum conjugate to $X_{k}^{I}, P^{I k}=2 \pi R T \frac{d X_{-k}^{I}}{d X^{0}}$. In quantum mechanics $a$ and $\tilde{a}$ become creation/annihilation operators, $\left[a^{k I}, a^{-k^{\prime} I^{\prime}}\right]=-k \delta_{k, k^{\prime}} \delta_{I, I^{\prime}}$. Let us consider the states of the form:

$$
\left(a^{k_{1} I_{1}}\right)^{n_{1}} \cdots\left(a^{k_{p} I_{p}}\right)^{n_{p}} \mid 0>
$$

These states are annihilated by $\tilde{a}^{k I}$ with negative $k$. Quasiclassically this means that the state is purely left moving. These are the BPS states. The classical BPS configurations given by (7) are superpositions of the large numbers of these quantum BPS states.

Suppose that we start increasing the string coupling constant. When $g_{s t r} \rightarrow \infty$ we can describe our D-string as a fundamental string of the dual Type IIB. In this limit the left-moving waves correspond to the BPS states in the spectrum of the fundamental string which have been discovered by Dabholkar and Harvey [13]. These states are the form

$$
\begin{aligned}
& \left(\alpha_{-k_{1}}^{I_{1}}\right)^{n_{1}} \cdots\left(\alpha_{-k_{p}}^{I_{p}}\right)^{n_{p}} \mid w_{9}, p_{9}> \\
& \sum k_{p} n_{p}=w_{9} p_{9}
\end{aligned}
$$

where $p_{9}$ is related to the momentum $P_{9}=\frac{1}{R} p_{9}$ and $w_{9}$ is the winding number; in our case $w_{9}=1$. The degeneracy of these states is $\mathcal{N}\left(p_{9}\right)$ - the coefficient of $q^{p_{9}}$ in $\prod_{m=1}^{\infty} \frac{1}{\left(1-q^{m}\right)^{8}}$ (in fact there is more degeneracy because of the worldsheet fermions). The number of states with the given momentum $P_{9}$ is finite although rapidly growing with $P_{9}$.

In this example, the degeneracy of states with the given momentum did not depend on $T R^{2}$. This is because the theory on the worldvolume was free. If we have added interactions, for example consider the D string living in the curved space then the spectrum would depend on $T r^{2}$ where $r$ determines the curvature. If in addition these interactions were nonrenormalizable then the spectrum would also depend on the cutoff (that is on $\left.\alpha^{\prime}\right)$.

\section{Supersymmetry for $\frac{1}{8}$ BPS giant gravitons in $A d S_{5} \times S^{5}$}

\subsection{Spinors in $A d S_{p-1} \times S^{q-1}$}

Spinors in the spaces of the form AdS times a sphere were studied in many papers 15, [16, 17, 18]. In this subsection we will briefly review this subject. 
First of all, let us remember what is the spin bundle. Consider a manifold $X$ with a metric $g_{\mu \nu}(x)$. Let us choose a field of tetrads $e^{1}(x), \ldots, e^{N}(x)$, where $N=\operatorname{dim} X$ and $g_{\mu \nu}=e_{\mu}^{m} e_{\nu}^{m}$, summation over $m$. A section of the spinor bundle $S(T X)$ is defined with respect to the choice of the field of tetrads; it is a field $\psi(x) \in \mathcal{S}$ where $\mathcal{S}$ is a spinor representation of $S O(N)$. When we change the field of tetrads $\psi(x)$ should change to $\rho_{s}(g(x)) \psi(x)$, where $g(x)$ is the unique element of $S O(N)$ such that the new tetrad is $e^{\prime}(x)=g(x) e(x)$ and $\rho_{s}(g(x))$ is the spinor representation of $g(x)$. There is a sign ambiguity in the choice of the spin representation, but it is not very important for us now. $S(T X)$ comes equipped with the connection, which is defined as follows. The section of the spinor bundle is covariantly constant along the path $x(\tau)$ if and only if $\psi(x)$ is constant provided that we have chosen the field of tetrads in such a way that $\frac{D}{D \tau} e^{m}(x(\tau))=0$. This connection is called "spin connection". It can be described in the following way. Let us choose $N$ constant gamma-matrices $\Gamma^{m}$, satisfying $\Gamma^{m} \Gamma^{n}+\Gamma^{n} \Gamma^{m}=2 \delta^{m n}$. Then,

$$
\nabla_{\mu} \psi(x)=\left(\partial_{\mu}+\frac{1}{4} \omega_{\mu}^{m n}\left[\Gamma^{m}, \Gamma^{n}\right]\right) \psi
$$

where $\omega^{m n}$ is a one-form determined from $d e^{m}=\omega^{m n} \wedge e^{n}$.

Suppose that we have an embedding of manifolds $i: X \subset Y, \operatorname{dim} X=k, \operatorname{dim} Y-$ $\operatorname{dim} X=l, l$ is even, both $X$ and $Y$ have a spin structure. The structure group of $i^{*}(T Y)$ is $\operatorname{Spin}(k) \times \operatorname{Spin}(l)$. In other words, we can define the field of tetrads in such a way that the first $k$ vectors $e_{m}$ belong to $T X$ and the last $l$ of them belong to $(T X)^{\perp}$. This gives us an isomorphism

$$
R: \quad S(T X) \otimes S\left((T X)^{\perp}\right) \subsetneq i^{*} S(T Y)
$$

However, one should remember that this isomorphism usually does not commute with the parallel transport.

It is important for us to consider a special case with $Y=N_{1} \times N_{2}, X=M_{1} \times M_{2}$, $M_{i} \subset N_{i}, \operatorname{dim} N_{i}-\operatorname{dim} M_{i}=1$. For example, we can take $N=\mathbf{R}^{2+4} \times \mathbf{R}^{6}$ and $M=$ $A d S_{5} \times S^{5}$. More generally, we assume that $N_{i}$ is the cone with the base $M_{i}$. This means that the metric on $N_{i}$ can be written as follows:

$$
d s_{M_{i}}^{2}=\left(\frac{d R}{R}\right)^{2}+e^{2 f(R)} d s_{N_{i}}^{2}
$$

We can write a general expression for some components of the spin connection:

$$
\omega^{i R}=-\frac{\partial f}{\partial R} e^{i}
$$

where $e^{i}$ is tangent to $M_{i}$. Suppose that $\Psi_{++}$is a covariantly constant spinor in $N_{1} \times N_{2}$ satisfying $\Gamma^{1 \ldots p} \Psi_{++}=-i^{\frac{p}{2}} \Psi$ and $\Gamma^{p+1 \ldots p+q} \Psi_{++}=-i^{\frac{q}{2}} \Psi$. Then, the restriction of the 
covariantly constant spinor to $M_{1} \times M_{2}$ satisfies the following equations:

$$
\begin{gathered}
\left(\frac{D}{D x^{\mu}}-i \frac{p}{2} \frac{\partial f}{\partial R} \Gamma^{\mu} \prod_{I=1}^{p-1} \Gamma^{I}\right) \Psi_{++}=0 \quad(\mu=1, \ldots, p-1) \\
\left(\frac{D}{D y^{\mu}}-i \frac{q}{2} \frac{\partial f}{\partial R} \Gamma^{p+\mu} \prod_{I=1}^{q-1} \Gamma^{p+I}\right) \Psi_{++}=0 \quad(\mu=1, \ldots, q-1)
\end{gathered}
$$

where $x^{\mu}$ are the coordinates on $M_{1}$ and $y^{\mu}$ are the coordinates on $M_{2}$. These are the equations for covariantly constant spinors in $A d S_{5} \times S^{5} . \Psi_{++}$is not the only solution to the equations (22). One can see that $\Gamma^{p} \Gamma^{p+q} \Psi_{++}$is another solution. In fact, our spinor representation is reducible if considered as a representation of $\Gamma^{1}, \ldots, \Gamma^{p-1}, \Gamma^{p+1}, \ldots, \Gamma^{p+q-1}$. The operator $\frac{1}{2}\left(1+i \Gamma^{p} \Gamma^{p+q}\right)$ is the projector on the irreducible subspace. This gives us the following description of the Killing spinors in $A d S_{p-1} \times S^{q-1}$ :

$$
\Psi=\frac{1}{2}\left(1+i \Gamma^{p} \Gamma^{p+q}\right) \Psi_{++}
$$

Here we have assumed that both the AdS and the sphere have Euclidean signature. The formula for the Minkowski AdS times the Euclidean sphere is the same except for one should skip $i$.

\subsection{Some geometric background}

First of all, let us summarize already introduced notations and introduce some more. We have embedded $M \subset N$, $\operatorname{dim} N$ - $\operatorname{dim} M=1, N$ has a complex structure $I$, which is an operator in $T N$ with $I^{2}=-1$ satisfying certain integrability conditions. For our application, we can take $M=S^{5}$ and $N=\mathbf{C}^{3}$, or $M=T^{1,1}$ and $N=$ conifold. We are considering a holomorphic surface $C \subset N$, $\operatorname{dim}_{\mathbf{C}} C=\operatorname{dim}_{\mathbf{C}} N-1$. In this situation, it is natural to consider the following obects:

\begin{tabular}{|c|l|}
\hline$e^{\perp}$ & Unit vector orthogonal to $T M \subset T N$ \\
\hline$e^{\|}$ & $I . e^{\perp}$ \\
\hline$\Sigma$ & $C \cap M$ \\
\hline$(T C)^{\perp}$ & Orthogonal complement of $T C$ in $T N$ \\
\hline$e^{\phi}$ & Unit vector in $(T C)^{\perp} \cap T M ;$ notice that $e^{\phi} \perp T \Sigma$ \\
\hline$T_{0} \Sigma$ & Maximal subspace in $T \Sigma$ closed under $I, I . T_{0} \Sigma=T_{0} \Sigma$ \\
\hline$e^{\psi}$ & Unit vector in the orthogonal complement to $T_{0} \Sigma$ in $T \Sigma$ \\
\hline$e^{n}$ & Unit vector in $T M$ orthogonal to $e^{\phi}$ and $T \Sigma$ \\
\hline
\end{tabular}

Notice that the orthogonal complement to $T \Sigma$ in $T M$ has real dimension two. In this subsection we will prove that the component of $e^{\| l}$ orthogonal to $T \Sigma$ is proportional to $e^{\phi}:$

$$
e^{\|}=\lambda e^{\phi} \bmod T \Sigma, \lambda \leq 1
$$

We will also find that the component of $e^{\| l}$ parallel to $T \Sigma$ is proportional to $e^{\psi}$ (in other words, it is orthogonal to $T_{0} \Sigma$ ). 
Consider I.e $e^{\phi}$. This vector should be orthogonal to $e^{\phi}$, and also orthogonal to $\Sigma$ (because $(T C)^{\perp}$ is closed under $I$ ). This means that the three vectors $I . e^{\phi}, e^{\perp}$ and $e^{n}$ are linearly dependent:

$$
I . e^{\phi}=\cos \alpha e^{\perp}+\sin \alpha e^{n}
$$

Taking the scalar product of this equation with $e^{\| l}$, we find that $e^{\|}$is orthogonal to $e^{n}$. This proves our first statement: the component of $e^{\|}$orthogonal to $T \Sigma$ is directed along $e^{\phi}$. Suppose that $v \in T_{0} \Sigma$. Then, $\left(e^{\|} \cdot v\right)=-\left(e^{\perp} \cdot I . v\right)=0$ because $I . v$ also belongs to $T_{0} \Sigma$. Therefore, $e^{\|}$is orthogonal to $T_{0} \Sigma$, which is our second statement. Now, we have $e^{\| l}$ a linear combination of $e^{\phi}$ and $e^{\psi}$ :

$$
e^{\|}=-\cos \beta e^{\phi}+\sin \beta e^{\psi}
$$

Applying $I$ to (25) we see that $I . e^{n}$ is also a linear combination of $e^{\phi}$ and $e^{\psi}$. The coefficients are partially fixed by $\left(I \cdot e^{n} \cdot e^{\| l}\right)=0$.

$$
I . e^{n}=\mp\left(\sin \beta e^{\phi}+\cos \beta e^{\psi}\right)
$$

Comparing this with (25) we find $\beta= \pm \alpha$.

We have proven the following statement: the normal component of $e^{\| l}$ is $-\cos \alpha e^{\phi}$, where $\cos \alpha$ is the coefficient in the linear dependence (25).

\subsection{Supersymmetry for $\frac{1}{8}$ BPS giant gravitons}

We will use an embedding

$$
A d S_{5} \times S^{5} \subset \mathbf{R}^{2+4} \times \mathbf{R}^{6}
$$

We enumerate gamma-matrices in such a way that $\Gamma^{5}$ corresponds to the direction orthogonal to $A d S_{5}$ in $\mathbf{R}^{2+4}$ and $\Gamma^{11}$ corresponds to the direction in $\mathbf{R}^{6}$ orthogonal to $S^{5}$. We will concentrate on the graviton spread inside $S^{5}$. We will think of $S^{5}$ as $M$ and $\mathbf{R}^{6}=\mathbf{C}^{3}$ as $N$. Given a vector $v$, we denote $\Gamma(v)$ the corresponding gamma-matrix. Combining these notations with the notations from the previous paragraph we can write $\Gamma^{11}=\Gamma\left(e^{\perp}\right)$. Our sign conventions imply $\left(\Gamma^{0}\right)^{2}=\left(\Gamma^{5}\right)^{2}=1$ and the other $\Gamma^{I}$ have $\left(\Gamma^{I}\right)^{2}=-1$. The covariantly constant spinor in $A d S_{5} \times S^{5}$ has a form

$$
\Psi=\left(1+\Gamma^{5} \Gamma^{11}\right) \Psi_{++}
$$

where $\Psi_{++}$is a constant spinor subject to the following constraints:

$$
\begin{gathered}
\Gamma^{0} \cdots \Gamma^{5} \Psi_{++}=i \Psi_{++} \\
\Gamma^{6} \cdots \Gamma^{11} \Psi_{++}=i \Psi_{++}
\end{gathered}
$$

Let $\Gamma^{\tau}$ corresponds to the direction of the timelike geodesic in $A d S_{5}$. The condition for preserved supersymmetry is

$$
\frac{1}{\sqrt{1-v^{2}}}\left(\Gamma^{\tau}+v \Gamma^{\phi}\right) \hat{\Sigma} \Psi=i \Psi
$$


Here $\Psi=\Psi_{L}+i \Psi_{R}$ is a combination of the Majorana-Weyl generators of supersymmetry in Type IIB. $\hat{\Sigma}=\frac{1}{6} \Sigma_{\alpha \beta \gamma} \Gamma^{\alpha} \Gamma^{\beta} \Gamma^{\gamma}$ is the gamma-matrix representation of the surface element of the $D 3$ brane. $\Gamma^{\phi}=\Gamma\left(e^{\phi}\right)$ is the gamma-matrix corresponding to the vector $e^{\phi}$ introduced in the previous section. Let us parametrize the velocity in terms of the angle $\nu$ : $v=\cos \nu$. We can rewrite the supersymmetry condition as follows:

$$
\begin{gathered}
0=\left[i\left(\Gamma^{\tau}+\cos \nu \Gamma^{\phi}\right) \hat{\Sigma}+\sin \nu\right]\left(1+\Gamma^{5} \Gamma^{11}\right) \Psi_{++}= \\
=\left(1+\Gamma^{5} \Gamma^{11}\right)\left[\Gamma^{\tau} \Gamma^{5} \Gamma^{n} \Gamma^{\phi}-\cos \nu \Gamma^{n} \Gamma^{11}+\sin \nu\right] \Psi_{++}
\end{gathered}
$$

Let us impose the following constraint on $\Psi_{++}$:

$$
\Gamma^{\tau} \Gamma^{5} \Gamma\left(e^{\phi}\right) \Gamma\left(I e^{\phi}\right) \Psi_{++}=\Psi_{++}
$$

Now we can see that the supersymmetry condition is satisfied for $\nu=-\alpha$ :

$$
\left(1+\Gamma^{5} \Gamma^{11}\right)\left[\Gamma^{n} \Gamma\left(I e^{\phi}\right)-\cos \alpha \Gamma^{n} \Gamma^{11}-\sin \alpha\right] \Psi_{++}=0
$$

which is a consequence of (25).

The constraint (32) is obviously compatible with (29). We can construct a spinor satisfying this constraint in the following way. Let us consider $\Psi_{++}$annihilated by all the gamma-matrices corresponding to the holomorphic directions in $\mathbf{C}^{3}$, and belonging to the $+i$-eigenspace of $\Gamma^{\tau} \Gamma^{5}$ :

$$
\begin{aligned}
& \Gamma\left(\partial_{Z^{I}}\right) \Psi_{++}=0, \quad I=1,2,3 \\
& \Gamma^{\tau} \Gamma^{5} \Psi_{++}=i \Psi_{++}
\end{aligned}
$$

It makes sense to impose the constraint $\Gamma^{\tau} \Gamma^{5} \Psi_{++}=i \Psi_{++}$because $\Gamma^{\tau} \Gamma^{5}$ is constant. Indeed, the timelike geodesics in $A d S_{5}$ are intersections with the $2+0$-dimensional planes in $\mathbf{R}^{2+4}$ passing through the origin. Therefore, $\Gamma^{\tau} \Gamma^{5}$ is the gamma-matrix representation of the tangent bivector to such a plane, which is constant:

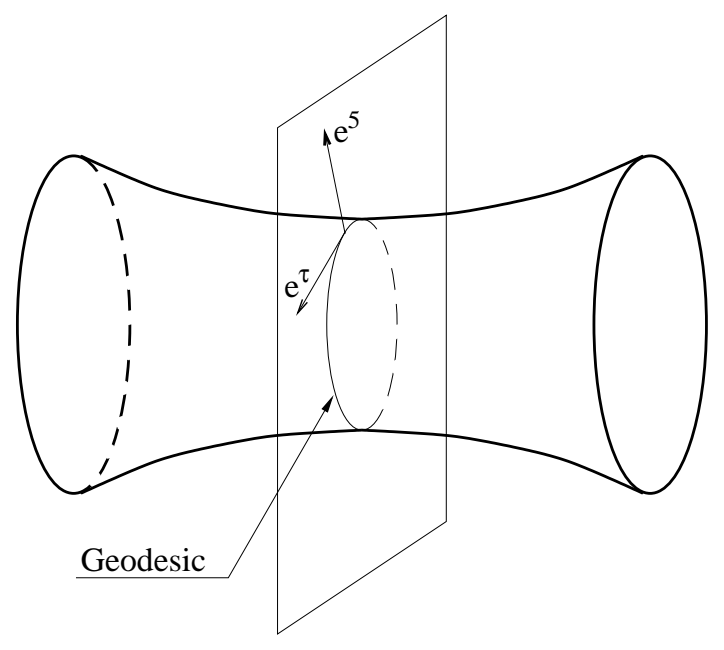

Timelike geodesic in AdS space 
Notice that the conditions (34) are generally speaking stronger then (32). Only $\frac{1}{8}$ of $\Psi_{++}$ satisfy them. For a class of $C$, we can find more spinors satisfying (32). Suppose that $C$ is described by $F=0$ where $F=F\left(Z_{1}, Z_{2}\right)$ depends only on the first two of the three $Z$. This means that $e^{\phi}$ and $I . e^{\phi}$ belong to $\mathbf{C}^{2} \subset \mathbf{C}^{3}$, the subset of $\mathbf{C}^{3}$ parametrized by $Z_{1}$ and $Z_{2}$. In this case, besides (34), we can also solve (32) as follows:

$$
\begin{aligned}
& \Gamma\left(\partial_{\bar{Z}^{1}}\right) \Psi_{++}=\Gamma\left(\partial_{\bar{Z}^{2}}\right) \Psi_{++}=0, \quad \Gamma\left(\partial_{Z^{3}}\right) \Psi_{++}=0 \\
& \Gamma^{\tau} \Gamma^{5} \Psi_{++}=-i \Psi_{++}
\end{aligned}
$$

Therefore, in this case we will have $\frac{1}{4}$ supersymmetries preserved. The giant graviton of [8] preserves $\frac{1}{2}$ supersymmetry, and it is natural to think of it as given by $F\left(Z_{1}\right)=0, F$ depends only on $Z_{1}$.

\section{Supersymmetry for $\frac{1}{4}$ BPS giant gravitons in $A d S_{3} \times S^{3}$}

Let us consider Type IIB compactified on $\mathbf{T}^{4}$, and wrap $N D 3$ branes on a two-cycle $c$ in $\mathbf{T}^{4}$. The near horizon geometry is $A d S_{3} \times S^{3}$. Let $\alpha^{\vee}$ be a constant two-form on $\mathbf{T}^{4}$ representing the Poincare dual to $c$ and $\alpha_{I J}=\frac{1}{2} \epsilon_{I J K L}\left(\alpha^{\vee}\right)^{K L}$. Then the five-form will have the following structure:

$$
F=\operatorname{vol}_{A d S_{3}} \wedge \alpha+\operatorname{vol}_{S^{3}} \wedge \alpha^{\vee}
$$

$\mathbf{T}^{4}$ has a sphere worth of complex structures; let us choose the one in which $c$ is represented by a holomorphic cycle. We will denote this complex structure $I$. Consider the embedding $A d S_{3} \times S^{3} \times \mathbf{T}^{4} \subset \mathbf{R}^{2+2} \times \mathbf{R}^{0+4} \times \mathbf{T}^{4}$. We denote the coordinates in $\mathbf{R}^{2+2} X^{0}, \ldots, X^{3}$, the coordinates in $\mathbf{R}^{0+4} X^{5}, \ldots, X^{7}$, and the coordinates in $\mathbf{T}^{4} X^{8}, \ldots, X^{11}$. The Killing spinors on $A d S_{3} \times S^{3}$ are parametrized by a constant spinor $\Psi_{+++}$in $\mathbf{R}^{2+10}$ which satisfies the following constraints:

$$
\begin{array}{r}
\Gamma^{8} \Gamma^{9} \Gamma^{10} \Gamma^{11} \Psi_{+++}=\Psi_{+++} \\
\Gamma^{0} \Gamma^{1} \Gamma^{2} \Gamma^{3} \Psi_{+++}=\Gamma(v) \Gamma(I . v) \Psi_{+++} \\
\Gamma^{4} \Gamma^{5} \Gamma^{6} \Gamma^{7} \Psi_{+++}=i \Gamma(v) \Gamma(I . v) \Psi_{+++}
\end{array}
$$

where $v$ is an arbitrary vector in $T \mathbf{T}^{4}$ (if (38) and (39) is true for some $v$, then it is true for any $v$ because of (37).) Such a $\Psi_{+++}$is an eigenvector of $\alpha_{I J} \Gamma^{7+I} \Gamma^{7+J}$ as well as of $\left(\alpha^{\vee}\right)_{I J} \Gamma^{7+I} \Gamma^{7+J}$. Given $\Psi_{+++}$satisfying $(37-39)$, the Killing spinor is

$$
\Psi=\left(1+\Gamma^{3} \Gamma^{7}\right) \Psi_{+++}
$$

Let us probe the near-horizon background with the brane of the same kind: the D3 wrapped on $c$. The supersymmetry condition is:

$$
\begin{aligned}
0 & =\left[\left(\Gamma^{\tau}+\cos \nu \Gamma^{\phi}\right) \Gamma^{\psi}+\sin \nu\right]\left(1+\Gamma^{3} \Gamma^{7}\right) \Psi_{+++}= \\
& =\left(1+\Gamma^{3} \Gamma^{7}\right)\left[-\Gamma^{\psi}\left(\Gamma^{\tau}+\cos \nu \Gamma^{\phi}\right)+\sin \nu\right] \Psi_{+++}
\end{aligned}
$$


where $\Gamma^{\psi}$ is the gamma-matrix corresponding to $e^{\psi}$ - the unit vector tangent to the black string. Just as in the previous section, we have

$$
e^{\phi}=-\frac{1}{\cos \beta} e^{\|}+\frac{\sin \beta}{\cos \beta} e^{\psi}
$$

- now this is just the statement that $e^{\phi}$ is the projection of $e^{\|}$on the space orthogonal to the black string. Let us introduce a complex structure $J$ in $\mathbf{R}^{4}$ in such a way that

$$
e^{\|}=J \cdot e^{\perp}
$$

We impose the following conditions on $\Psi_{+++}$:

$$
\begin{gathered}
\Gamma^{4} \Gamma^{5} \Gamma^{6} \Gamma^{7} \Psi_{+++}=\Psi_{+++} \\
\Gamma^{\tau} \Gamma^{3} \Gamma^{\| l} \Gamma^{7} \Psi_{+++}=-\Psi_{+++}
\end{gathered}
$$

If the second of these two conditions is satisfied at some point of $S^{3}$, then it is also satisfied at any other point. This follows from the first condition and from (43) (remember that in our notations $\Gamma\left(e^{\perp}\right)$ is the same as $\left.\Gamma^{7}\right)$.

One can see that (41) follows from (44). Therefore, the black string translated with the speed of light in the direction $e^{\|}$preserves $\frac{1}{4}$ of the supersymmetry independently of its shape.

\section{$5 \quad A d S_{4} \times S^{7}$ and $A d S_{7} \times S^{4}$}

To find supersymmetric configurations in $A d S_{4} \times S^{7}$ we embed $S^{7}$ into $\mathbf{C}^{4}$. Giant gravitons are intersections of $S^{7}$ with the holomorphic surfaces in $\mathbf{C}^{4}$. Spherical giant gravitons of [8] preserve $\frac{1}{2}$ of the supersymmetry. Gravitons of the form $F\left(Z_{1}, Z_{2}\right)=0$ preserve $\frac{1}{4}$ and those given by $F\left(Z_{1}, Z_{2}, Z_{3}\right)=0$ as well as $F\left(Z_{1}, Z_{2}, Z_{3}, Z_{4}\right)=0$ preserve $\frac{1}{8}$. The representation theory of $O \operatorname{sp}(8 \mid 4, \mathbf{R})$ admits also BPS multiplets preserving $\frac{3}{8}$ of the supersymmetry [19]. But we do not expect to find the $\frac{3}{8}$ BPS states in the spectrum of the superconformal theory on the boundary of $A d S_{4} \times S^{7}$ [20].

In $A d S_{7} \times S^{4}$ we should find $\frac{1}{2}$ and $\frac{1}{4}$ BPS configurations [19]. We construct the corresponding giant gravitons in the following way. Embed $S^{4} \subset \mathbf{R}^{5}$, and represent $\mathbf{R}^{5}=\mathbf{C}^{2} \times \mathbf{R}$. Consider three-dimensional cylinders in $\mathbf{C}^{2} \times \mathbf{R}$ of the form $C \times \mathbf{R}$ where $C \subset \mathbf{C}^{2}$ is a holomorphic curve. Let us denote $Z_{1}$ and $Z_{2}$ the coordinates in $\mathbf{C}^{2}$. The one-parametric group of symmetries $Z_{I} \rightarrow e^{i \phi} Z_{I}$ preserves the $S^{4}$. Let us denote $e^{\| l}$ the corresponding vector field on $S^{4}$. The normalization of $e^{\|}$is such that $\left\|e^{\|}\right\|^{2}=1$ on the equator of $S^{4}$. Consider $\Sigma=(C \times \mathbf{R}) \cap S^{4}$ the intersection of the tube $C \times \mathbf{R}$ with $S^{4}$. Let us think of $\Sigma$ as the worldvolume of the $M 2$ brane at time zero, and let us move it with the speed of light along $e^{\|}$. The corresponding brane configuration is supersymmetric.

Let us show that it is supersymmetric. We denote $e^{\phi}$ the component of $e^{\|}$orthogonal to $T \Sigma$ normalized to $\left\|e^{\phi}\right\|^{2}=1$ and $\Gamma^{\phi}$ the gamma-matrix representation of $e^{\phi}$. The 
perpendicular component of the velocity is of the magnitude $|\cos \nu|=\left|\left(e^{\|} \cdot e^{\phi}\right)\right|$. The supersymmetry condition has a form very similar to (31):

$$
\left(1+\Gamma^{4} \Gamma^{12}\right)\left[\Gamma^{\tau} \Gamma^{4} \Gamma^{n} \Gamma^{\phi}+\cos \nu \Gamma^{n} \Gamma^{12}-\sin \nu\right] \Psi_{+}=0
$$

Here $\Psi_{+}$is a constant Majorana-Weyl spinor in $\mathbf{R}^{2+10}$ satisfying the following constraint:

$$
\Gamma^{5} \cdots \Gamma^{12} \Psi_{+}=\Psi_{+}
$$

Let us denote $I$ the operator in $T \mathbf{R}^{5}=T\left(\mathbf{C}^{2} \times \mathbf{R}\right)$, which acts as zero on the vectors parallel to the $\mathbf{R}$-direction, and as a complex structure on the vectors parallel to $\mathbf{C}^{2}$. For any vector $v, I . v$ is parallel to $\mathbf{C}^{2}$. Notice that $e^{\phi}$ is orthogonal to the $\mathbf{R}$-direction in $\mathbf{C}^{2} \times \mathbf{R}$. (Let us explain why $e^{\phi}$ is orthogonal to $\mathbf{R}$. Since $e^{\| l}$ is orthogonal to $\mathbf{R}$, it is enough to show that the projection of $e^{\| l}$ on $\Sigma$ is orthogonal to $\mathbf{R}$. Let us denote $e^{\chi}$ the unit vector in $T \Sigma \cap \mathbf{R}^{\perp}$. Let us show that the projection of $e^{\|}$on $T \Sigma$ is in fact proportional to $e^{\chi}$. Indeed, $T \Sigma$ is generated by $e^{\chi}$ and the vector orthogonal to $e^{\chi}$. The vector orthogonal to $e^{\chi}$ is a linear combination of $I . e^{\chi}$ and the vector parallel to $\mathbf{R}$. But $e^{\|}$is orthogonal to $I . e^{\chi}$ because $I . e^{\|}$is orthogonal to $e^{\chi}$. Therefore, $e^{\|}$is orthogonal to the generator of $T \Sigma$ perpendicular to $e^{\chi}$, and thus the projection of $e^{\|}$on $T \Sigma$ is parallel to $e^{\chi}$.) In other words, both $e^{\phi}$ and $I . e^{\phi}$ are parallel to $\mathbf{C}^{2}$. Now let us impose the following conditions on $\Psi_{+}$:

$$
\Gamma^{\tau} \Gamma^{4} \Gamma(v) \Gamma(I . v) \Psi_{+}=\Psi_{+} \text {for any } v \in T \mathbf{C}^{2} \subset T\left(\mathbf{C}^{2} \times \mathbf{R}\right),\|v\|^{2}=1
$$

Using this condition in (45) we get

$$
\left(1+\Gamma^{4} \Gamma^{12}\right)\left[\Gamma^{n} \Gamma\left(I . e^{\phi}\right)+\cos \nu \Gamma^{n} \Gamma^{12}-\sin \nu\right] \Psi_{+}=0
$$

Notice that there is a relation of the form

$$
I . e^{\phi}=\cos \alpha e^{\perp}+\sin \alpha e^{n}
$$

with some $\alpha$. Here $e^{\perp}$ denotes a vector orthogonal to $S^{4}$ in $\mathbf{R}^{5}$ (in our notations it is the same as $e^{12}$ ) and $e^{n}$ denotes a vector in $T S^{4}$ orthogonal to both $e^{\phi}$ and $T \Sigma$. Indeed, the projection of $I . e^{\phi}$ to $S^{4}$ should be orthogonal to both $T \Sigma$ and $e^{\phi}$. (Let us explain why it is orthogonal to $T \Sigma$. The tangent space to $\Sigma$ is generated by $e^{\chi}$ and $I . e^{\chi}+w$ where $w$ is some vector in the $\mathbf{R}$-direction. We have to show that $I . e^{\phi}$ is orthogonal to $e^{\chi}$ and I. $e^{\chi}$. First of all, $\left(I . e^{\phi} \cdot e^{\chi}\right)=c\left(I . e^{\|} \cdot e^{\chi}\right)=0$ since $I . e^{\| l}$ is a sum of $e^{\perp}$ and a vector

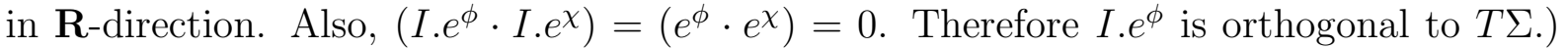
Therefore there is a relation of the form (49). This relation implies that (48) is satisfied provided that $\nu=\alpha+\pi$. (Remember that in our notations $\Gamma^{12}$ is the same as $\Gamma\left(e^{\perp}\right)$.) We need more work to verify that $\nu=\alpha+\pi$.

Let us denote $h$ the coordinate on $\mathbf{R}$, so that the equator of $S^{4}$ is at $h=0$ and the poles of $S^{4}$ are at $h= \pm 1$. For $|h|<1$ we introduce $\theta, h=\cos \theta$. Let us denote $E^{\|}=\frac{1}{\sin \theta} e^{\|}$. 
We have $\left\|E^{\|}\right\|^{2}=1$. For any $h_{0} \in \mathbf{R}$ we can consider the plane $H_{h}$ consisting of the points with $h=h_{0}$. We denote $S_{h}^{3}=H_{h} \cap S^{4}$ and $E^{\perp}$ the unit vector normal to $S_{h}^{3}$ in $H_{h}$. Now $E^{\| l}$ can be decomposed as follows:

$$
E^{\|}=-\cos \beta e^{\phi}+\sin \beta e^{\chi}
$$

(This is just a statement that the projection of $e^{\|}$on $T \Sigma$ is proportional to $e^{\chi}$ and the orthogonal projection is proportional to $e^{\phi}$.) This implies that

$$
\cos \nu=-\sin \theta \cos \beta
$$

Let us consider the intersection $\gamma=\Sigma \cap S_{h}^{3}$. This $\gamma$ is a string (real one-dimensional curve) in $S_{h}^{3}$. The trajectory of this string in $S_{h}^{3}$ is exactly the same as the motion of the D-string in the $S^{3}$ of $A d S_{3} \times S^{3}$ considered in Section 4. Let $E^{n}$ be the unit vector in $T S_{h}^{3}$ orthogonal to both $T \gamma$ and $e^{\phi}$. The analysis similar to Section 3.2 shows that

$$
I . e^{\phi}=\cos \beta E^{\perp} \pm \sin \beta E^{n}
$$

where $\beta$ is the same $\beta$ as in (50). Let us compare this relation with (49). Taking a scalar product of (49) and (52) with $e^{\perp}$ we get:

$$
\cos \alpha=\left(e^{\perp} \cdot E^{\perp}\right) \cos \beta=\sin \theta \cos \beta
$$

Now (51) and (53) imply that $\nu= \pm \alpha+\pi$ up to sign. This is what we needed (the sign can be adjusted by changing the orientation of $M 2$ ).

\section{Energy and Angular Momentum}

In this section we consider giant gravitons in $A d S_{5} \times S^{5}$.

\subsection{Energy of the Giant Graviton}

The energy is given by the following formula:

$$
E=\int_{\Sigma} d^{3} \sigma \frac{1}{\sqrt{1-v_{\perp}^{2}}}=\int_{\Sigma} d^{3} \sigma \frac{1}{\left|\left(e^{\|} \cdot e^{\psi}\right)\right|}=\int_{\Sigma} d^{3} \sigma \frac{1}{\left|\left(e^{\perp} \cdot I . e^{\psi}\right)\right|}
$$

Notice that $\left(e^{\perp} \cdot I . e^{\psi}\right)$ is the cosine of the angle between $C$ and the normal vector to $S^{5}$. This enables us to express the integral as follows:

$$
E=\int_{C} d \operatorname{vol}(C) \delta\left(\sqrt{\left|Z_{1}\right|^{2}+\left|Z_{2}\right|^{2}+\left|Z_{3}\right|^{2}}-1\right)
$$

This is the mass formula. 


\subsection{BPS bound}

Here we will express the energy of the giant graviton in terms of its momentum. Let us remember the definition of the momentum. Consider a classical mechanical system with the action being invariant under the global symmetry. This means that when we vary the trajectory $\delta \phi^{i}(t)=\epsilon \xi^{i}(\phi(t))$ with $\xi^{i}$ being the generator of the symmetry, the action does not change:

$$
\delta_{\epsilon} S=\epsilon \int d t \xi^{i}(\phi(t)) \frac{\delta S}{\delta \phi^{i}(t)}=0
$$

Suppose now that $\epsilon$ depends on $t$. Then, the variation of the action is:

$$
\delta_{\epsilon(t)} S=\int d t \dot{\epsilon}(t) M(\phi(t))
$$

where $M(\phi)$ is the momentum. On the classical trajectories, the variation of the action is zero anyway; this implies that $\dot{M}(\phi(t))=0$ - the momentum conservation.

In our situation the action is invariant under $S O(6)$. Consider a subgroup $U(1)_{I} \subset$ $S O(6)$ generated by the overall phase rotations $Z_{I} \rightarrow e^{i t} Z_{I}, I=1,2,3$. Let $M$ denote the corresponding momentum. The corresponding infinitesimal symmetry is $\delta Z_{I}=i \epsilon Z_{I}$. Let us compute $M$. The Born-Infeld action consists of two terms, $\int \sqrt{\operatorname{det} \partial X \cdot \partial X}$ and the Wess-Zumino term $S_{W Z}$. We will denote these contributions $M_{0}$ and $M^{\prime}$, respectively. Let us start with calculating $M_{0}$ :

$$
M_{0}=\int_{\Sigma} d \sigma \frac{v_{\perp}^{2}}{\sqrt{1-v_{\perp}^{2}}}=\int_{\Sigma} d \sigma\left(\frac{1}{\left(e^{\|} \cdot e^{\psi}\right)}-\left(e^{\|} \cdot e^{\psi}\right)\right)
$$

This looks like energy, except for the second term, $-\int_{\Sigma} d^{3} \sigma\left(e^{\|} \cdot e^{\psi}\right)$. We want to show that this term cancels by the contribution from the Wess-Zumino term. Indeed, the contribution of the Wess-Zumino term to the angular momentum can be written as follows:

$$
\Delta M=\int_{\partial^{-1 \Sigma}} \iota\left(e^{\|}\right) F
$$

where $\partial^{-1} \Sigma$ is a surface with the boundary $\Sigma$, and $F$ is the five-form, which is proportional to the volume form ${ }^{5}$ on $S^{5}$. One can write $F=4 \iota\left(e^{\perp}\right) \cdot \frac{1}{6} \omega \wedge \omega \wedge \omega$ where $\omega$ is a Kahler form on $\mathbf{C}^{3}$. Also, notice that for the restriction on the tangent space to $S^{5}$, we have:

$$
\left.\left(\iota\left(e^{\|}\right) . \iota\left(e^{\perp}\right) \cdot \omega \wedge \omega \wedge \omega\right)\right|_{T S^{5}}=\left.3(\omega \wedge \omega)\right|_{T S^{5}}
$$

\footnotetext{
${ }^{2}$ The coefficient of proportionality for $A d S_{5} \times S^{5}$ is 4 . One can remember it in the following way. Consider the spherical $D 3$ brane in $A d S_{5}$ approaching the boundary at $r=\infty$. The leading contributions to the action from the Born-Infeld term and the action should cancel when $r \rightarrow \infty$ [21]. Suppose that we change $r$ a little bit. The Born-Infeld part of the action will change as $\int d t \delta\left(r^{4} \operatorname{vol}\left(S^{3}\right)\right)$ (we are using the metric $-\left(1+r^{2}\right) d t^{2}+\frac{d r^{2}}{1+r^{2}}+r^{2} d s_{S^{3}}^{2}$ on $\left.A d S_{5}\right)$. The change in the Wess-Zumino coupling will be $C \int d t \delta r r^{3} \operatorname{vol}\left(S^{3}\right)$ where $C$ is the coefficient of proportionality between $F_{5}$ and the volume form. Therefore $C=4$.
} 
(this is because $\left.\iota\left(e^{\|}\right) \cdot \omega\right|_{T S^{5}}=0$.) Let us call $C_{+}$the part of $C$ which is inside $S^{5}$ and $C_{-}$ the part of $C$ which is outside $S^{5}$. Using $d(\omega \wedge \omega)=0$ we can rewrite (59) as follows:

$$
\Delta M=2 \int_{C_{+}} \omega \wedge \omega
$$

Now let us use the fact that

$$
\omega=\frac{1}{4} d \sum_{I=1}^{3}\left(\bar{Z}^{I} d Z^{I}-Z^{I} d \bar{Z}^{I}\right)
$$

This allows us to write

$$
\Delta M=\frac{1}{2} \int_{\partial C_{+}=\Sigma} \omega \wedge \sum_{I=1}^{3}\left(\bar{Z}^{I} d Z^{I}-Z^{I} d \bar{Z}^{I}\right)
$$

One can see that $\left.\omega\right|_{\Sigma}$ is the area for on $T_{0} \Sigma$ and

$$
\sum_{I=1}^{3}\left(\bar{Z}^{I} d Z^{I}-Z^{I} d \bar{Z}^{I}\right)=2\left(e^{\perp} \cdot I . e^{\psi}\right) d \psi
$$

where $d \psi$ is the length form on the orthogonal complement to $T_{0} \Sigma$ in $T \Sigma$. Therefore,

$$
\Delta M=\int_{\Sigma} d^{3} \sigma\left(e^{\perp} \cdot I . e^{\psi}\right)=\int_{\Sigma} d^{3} \sigma\left(e^{\|} \cdot e^{\psi}\right)
$$

which is what we wanted to prove. We see that the energy of the giant graviton is equal to the value of the angular momentum on the generator of $U(1)_{I} \subset S O(6)$. This is the BPS bound.

\subsection{Some examples}

We have not computed the angular momentum of the giant graviton in the closed form. However, for some giant gravitons we can predict the direction of the angular momentum. Let us consider the giant graviton corresponding to the following surface $C$ :

$$
Z_{1}^{a_{1}} Z_{2}^{a_{2}} Z_{3}^{a_{3}}=\rho
$$

The worldvolume of this giant graviton is invariant under $U(1)^{3} \subset S O(6)$. The first $U(1)$, which we will call $U(1)_{I}$, is just $Z_{I} \rightarrow e^{i t} Z_{I}$. The second and the third $U(1)$, which we will call $U(1)_{\left(a_{1}, a_{2}, a_{3}\right)}^{2}$, is $Z_{I} \rightarrow e^{i s w_{I}} Z_{I}$ where $\sum_{I=1}^{3} w_{I} a_{I}=0$. These three $U(1)$ generate the maximal torus of $S O(6)$. Since the worldvolume is invariant under $U(1)^{3}$, the angular momentum should commute with $U(1)^{3}$. Therefore it belongs to the Cartan subalgebra. One can also see that the angular momentum is orthogonal to the generators 
of $U(1)_{\left(a_{1}, a_{2}, a_{3}\right)}^{2}$. In our situation, generators of $U(1)_{\left(a_{1}, a_{2}, a_{3}\right)}^{2}$ act as reparametrizations on the worldvolume. Since the Born-Infeld action for the D3 brane is reparametrization invariant, it follows that $\delta_{\epsilon} S$ is zero independently of whether or not $\epsilon$ depends on $t$. Therefore, the component of the angular momentum corresponding to $U(1)_{\left(a_{1}, a_{2}, a_{3}\right)}^{2}$ is zero. In fact, there is a subtlety here. The Born-Infeld action includes the Wess-Zumino term, and we have to exercise care in defining itf. Given the worldsurface $\Sigma(t)$, let us consider the four-dimensional surface $\Lambda(t)$, such that its boundary is $\Sigma$ :

$$
\partial \Lambda(t)=\Sigma(t)
$$

The Wess-Zumino term in the action is the integral of the five-form field strength over the surface swept by $\Lambda(t)$. The contribution of the Wess-Zumino term to the angular momentum is zero provided that the symmetry acts as a reparametrization not only on $\Sigma$, but also on $\Lambda$. In our case, we can choose $\Lambda$ to be a family of surfaces starting with $\Sigma$, parametrized by $k, 1 \leq k<\infty$. This family is described by the equation:

$$
Z_{1}^{a_{1}} Z_{2}^{a_{2}} Z_{3}^{a_{3}}=k \rho, \quad 1 \leq k<\infty
$$

One can see that $U(1)_{\left(a_{1}, a_{2}, a_{3}\right)}^{2}$ preserves this family, therefore the contribution of the Wess-Zumino term to the $U(1)_{\left(a_{1}, a_{2}, a_{3}\right)}^{2}$ component of the momentum is zero. The angular momentum of our graviton is a linear combination of the generators of $U(1)_{I}$ and $U(1)_{\left(a_{1}, a_{2}, a_{3}\right)}^{2}$, orthogonal to the generators of $U(1)_{\left(a_{1}, a_{2}, a_{3}\right)}^{2}$. Therefore, it has the following form:

$$
M=\mu\left(\begin{array}{cccccc}
0 & -a_{1} & 0 & 0 & 0 & 0 \\
a_{1} & 0 & 0 & 0 & 0 & 0 \\
0 & 0 & 0 & -a_{2} & 0 & 0 \\
0 & 0 & a_{2} & 0 & 0 & 0 \\
0 & 0 & 0 & 0 & 0 & -a_{3} \\
0 & 0 & 0 & 0 & a_{3} & 0
\end{array}\right)
$$

Here we have used the basis $\left(\operatorname{Re} Z_{1}, \operatorname{Im} Z_{1}, \operatorname{Re} Z_{2}, \operatorname{Im} Z_{2}, \operatorname{Re} Z_{3}, \operatorname{Im} Z_{3}\right)$ in $\mathbf{R}^{6}$. The coefficient $\mu$ is a function of $\rho$.

\section{Supersymmetric branes in flat space}

It is interesting that the analogue of our construction works in flat space. The proof is actually simpler in flat space. In flat space we can get only noncompact BPS configurations (extending to the spatial infinity). We consider Type IIB in a flat ten-dimensional Minkowski space.

\footnotetext{
${ }^{3}$ I want to thank O. DeWolfe for discussions on the role of the Wess-Zumino term.
} 


\subsection{D1 brane}

Here we want to consider a well known example of a supersymmetric moving brane in Type IIB which is in some sense analogous to those configurations which we have constructed in AdS times sphere. The D1 brane of arbitrary shape moving with the speed of light in arbitrary direction $e^{\|}$preserves $\frac{1}{4}$ of the supersymmetry'. The preserved generators satisfy

$$
\Gamma^{0} \Gamma\left(e^{\|}\right) \psi=\psi, \quad \psi=i \psi^{*}
$$

Let us prove it. Suppose that

$$
e^{\|}=-\cos \alpha e^{\phi}+\sin \alpha e^{\psi}
$$

where $e^{\phi}$ is orthogonal to the worldline of the $D 1$ brane and $e^{\psi}$ belongs to the worldline. The supersymmetry preserved in the presence of a D string is $\Gamma^{0} \Gamma^{1} \psi=i \psi^{*}$. Using $\psi=i \psi^{*}$ we can rewrite this condition as follows:

$$
\frac{\Gamma^{0}-\cos \alpha \Gamma\left(e^{\phi}\right)}{\sin \alpha} \Gamma\left(e^{\psi}\right) \psi=\psi
$$

One can see that (67) and (68) implies (69).

\subsection{D3 brane}

Some examples of supersymmetric D3 branes in flat space can be obtained from the supersymmetric $D 1$ branes of the previous subsection. Their worldvolume is a product of a real one-dimensional curve $\gamma$ a complex curve $\beta$ which is orthogonal to $\gamma$. To preserve supersymmetry this brane should move with the speed of light in the direction orthogonal to $\beta$.

There are more general examples. Consider the five-dimensional plane $M \subset \mathbf{R}^{1+9}$. We will embed $M \subset \mathbf{C}^{3}$. Again, we have $e^{\|}$- the preferred direction in $T M, e^{\|}=I e^{\perp}$. Let $C$ be the complex surface in $\mathbf{C}^{3}, \Sigma=C \cap M$, and move $\Sigma$ with the speed of light in the direction $e^{\|}$. We denote $\cos \alpha e^{\phi}$ the projection of $e^{\| l}$ to the direction orthogonal to $T \Sigma$, and it turns out that $e^{\phi}$ is the unit vector in the intersection of $(T C)^{\perp}$ with $T M$, and the tangential component is proportional to $e^{\psi}$, just as in (26).

The supersymmetry condition:

$$
\left[i\left(\Gamma^{0}+\cos \alpha \Gamma\left(e^{\phi}\right)\right) \hat{\Sigma}+\sin \alpha\right] \psi=0
$$

Let us impose the following conditions on $\psi$ :

$$
\begin{aligned}
& \text { 1) } \Gamma^{0} \Gamma\left(e^{\|}\right) \psi=\psi, \\
& \text { 2) } \gamma(v) \gamma(I v) \psi=i\|v\|^{2} \psi, \text { for any } v \in T_{0} M
\end{aligned}
$$

\footnotetext{
${ }^{4}$ Suppose that at infinity the brane approaches a straight line parallel to $e^{\| l}$; in this case our configuration would be called "left-moving wave"; it preserves $\frac{1}{2}$ SUSY on the worldvolume theory of a D-string, and correspondingly $\frac{1}{4}$ SUSY of the target space theory.
} 
(we denote $T_{0} M$ the subspace of $T M$ orthogonal to $e^{\| l}$ ). Notice that from the second condition follows $\hat{\Sigma} \psi=i \Gamma\left(e^{\psi}\right) \psi$. Now we can rewrite the supersymmetry condition as follows:

$$
\begin{aligned}
& 0=\left[-\left(\Gamma^{0}+\cos \alpha \Gamma^{\phi}\right) \Gamma\left(e^{\psi}\right)+\sin \alpha\right] \psi= \\
& =\left[\Gamma\left(e^{\psi}\right)\left(\Gamma^{0}+\cos \alpha \Gamma\left(e^{\phi}\right)\right)+\sin \alpha\right] \psi= \\
& =\left[\Gamma\left(e^{\psi}\right)\left(\Gamma\left(e^{\|}\right)+\cos \alpha \Gamma\left(e^{\phi}\right)\right)+\sin \alpha\right] \psi
\end{aligned}
$$

which is the consequence of the first of Eqs. (26).

\section{A Giant gravitons in perturbation theory}

The authors of [12] have studied excitations around the spherical giant gravitons. They have used the quadratic approximation to the action and computed the frequencies of the normal modes. It was found that the excitations along AdS decouple in the quadratic approximation from the excitations along the sphere. Let us look at those excitations which are inside the sphere, which means $v_{k}=0$ in the notations of 12. Suppose that the sphere has dimension $n$. The frequencies are given by the formula (4.17 of [12]):

$$
\omega_{ \pm}^{2}=Q+\frac{(n-3)^{2}}{2} \pm(n-3) \sqrt{Q+\frac{(n-3)^{2}}{4}}
$$

where $Q$ is the eigenvalue of the Laplace operator on a sphere of dimension $n-2$ with the radius 1 . The possible values for $Q$ are $N(N+n-3)$ where $N$ is an integer. Therefore $\omega_{+}= \pm(N+n-3)$ and $\omega_{-}= \pm N$. The frequencies are integer, therefore the motion is periodic. The configuration returns to itself after its projection on AdS travels along the geodesic the distance equal to the circumference of the sphere. But this is exactly what we expect to see when we study in perturbation theory the $\frac{1}{4}$ or $\frac{1}{8}$ supersymmetric giant gravitons which are close to the given spherical configuration. Indeed, the flow generated by the vector field $e_{\|}$is periodic with the same period.

Most of the excitations found in [12] are not BPS. But some of them should be BPS and should represent our configurations in the linearized approximation.

\section{Acknowledgements}

I would like to thank O. DeWolfe, D. Gross, A. Hashimoto, S. Hirano, N. Itzhaki and E. Witten for illuminating discussions. This work was supported in part by the NSF Grant No. PHY99-07949, and in part by RFFI Grant No. 00-02-16477 and by the Russian grant for the support of the scientific schools No. 00-15-96557.

\section{References}


[1] J.M. Maldacena, "The Large N Limit of Superconformal Field Theories and Supergravity", Adv.Theor.Math.Phys. 2 (1998) 231-252; Int.J.Theor.Phys. 38 (1999) 1113-1133.

[2] S.S. Gubser, I.R. Klebanov, A.M. Polyakov, "Gauge Theory Correlators from NonCritical String Theory", Phys.Lett. B428 (1998) 105-114.

[3] E. Witten, "Anti De Sitter Space And Holography", Adv. Theor. Math. Phys. 2 (1998) 253-291.

[4] H.J. Kim, L.J. Romans, P. van Nieuwenhuizen, "The mass spectrum of chiral $\mathcal{N}=2$ $d=10$ supergravity on $S^{5}$ ", Phys. Rev. D32 (1985) 389.

[5] P. van Nieuwenhuizen, "The Complete Mass Spectrum of $d=11$ Supergravity Compactified on $S^{4}$ and a General Mass Formula for Arbitrary Cosets on $M_{4}$ ", Class. Quantum Grav. 2 (1985) 1.

[6] B. Biran, A. Casher, F. Englert, M. Rooman and P. Spindel, "The Fluctuating Seven Sphere in Eleven Dimensional Supergravity", Phys. Lett. B134 (1984) 179.

[7] L. Castellani, R. D'Auria, P. Fre, K. Pilch, P. Van Nieuwenhuizen, "The Bosonic Mass Formula for Freund-Rubin Solutions of $d=11$ Supergravity General Cosets Manifolds," Class. Quantum Grav. 1 (1984) 339.

[8] J. McGreevy, L. Susskind, N. Toumbas, "Invasion of the Giant Gravitons from Antide Sitter Space", hep-th/0003075.

[9] R.C. Myers, "Dielectric-Branes", hep-th/9910053, JHEP 9912 (1999) 022.

[10] M.T. Grisaru, R.C. Myers, O. Tafjord, "SUSY and Goliath", hep-th/0008015, JHEP 0008 (2000) 040.

[11] A. Hashimoto, S. Hirano, N. Itzhaki, "Large branes in AdS and their field theory dual", hep-th/0008016, JHEP 0008 (2000) 051.

[12] S. R. Das, A. Jevicki and S. D. Mathur, "Vibration modes of giant gravitons", hepth/0009019.

[13] A. Dabholkar, J.A. Harvey, "Nonrenormalization of the superstring tension", Phys. Rev. Lett. 63 (1989) 478.

[14] P. Candelas and X. de la Ossa, "Comments on conifolds", Nucl. Phys. B342 (1990) 246.

[15] C. Bär, "Real Killing spinors and holonomy", Comm. Math. Phys. 154 (1993) 509521. 
[16] I. Klebanov and E. Witten, "Superconformal Field Theory on Threebranes at a Calabi-Yau Singularity", Nucl.Phys. B536 (1998) 199-218, hep-th/9807080.

[17] Y. Imamura, "Supersymmetries and BPS Configurations on Anti-de Sitter Space", Nucl.Phys. B537 (1999) 184-202, hep-th/9807179.

[18] A. Kehagias, "New Type IIB Vacua and their F-Theory Interpretation", Phys.Lett. B435 (1998) 337-342, hep-th/9805131.

[19] S. Ferrara, E. Sokatchev, "Conformal superfields and BPS states in $A d S_{4 / 7}$ geometries", hep-th/0007058.

[20] S. Ferrara, E. Sokatchev, "Superconformal interpretation of BPS states in AdS geometries", hep-th/0005151.

[21] N. Seiberg, E. Witten, "The D1/D5 System And Singular CFT", JHEP 9904 (1999) 017, hep-th/9903224. 